\title{
Grapheme-color synesthetes show peculiarities in their emotional brain: cortical and subcortical evidence from VBM analysis of 3D-T1 and DTI data
}

\author{
Helena Melero • Ángel Peña-Melián • Marcos Ríos-Lago • \\ Gonzalo Pajares · Juan Antonio Hernández-Tamames • \\ Juan Álvarez-Linera
}

\begin{abstract}
Grapheme-color synesthesia is a neurological phenomenon in which viewing achromatic letters/numbers leads to automatic and involuntary color experiences. In this study, voxel-based morphometry analyses were performed on T1 images and fractional anisotropy measures to examine the whole brain in associator grapheme-color synesthetes. These analyses provide new evidence of variations in emotional areas (both at the cortical and subcortical levels), findings that help understand the emotional component as a relevant aspect of the synesthetic experience. Additionally, this study replicates previous findings in the left intraparietal sulcus and, for the first time, reports the existence of anatomical differences in subcortical gray nuclei of developmental grapheme-color synesthetes, providing a link between acquired and developmental synesthesia. This empirical evidence, which goes beyond modality-specific areas, could lead to a better understanding of
\end{abstract}

grapheme-color synesthesia as well as of other modalities of the phenomenon.

Keywords Grapheme-color synesthesia - Voxel-based morphometry · Cortical emotional areas - Subcortical gray nuclei
Abbreviation
GC Grapheme-color synesthesia
VBM Voxel-based morphometry
DTI Diffusion tensor imaging
DARTEL Diffeomorphic anatomical registration through exponentiated lie algebra method
FA Fractional anisotropy
FG Fusiform gyrus
IPS Intraparietal sulcus
TMS Transcranial magnetic stimulation
ORS Oscillatory resonance supervenience model
PHg Parahippocampal gyrus
TP Temporal pole 
aIN Anterior insula

RSC Retrosplenial cortex

ACG Anterior cingulate gyrus

MdFG Middle frontal gyrus

\section{Introduction}

Synesthesia-from the ancient Greek ov́v (syn), "together," and $\alpha \ddot{i} \sigma \theta \eta \sigma ı \varsigma$ (aisthessis), "sensation"-is a perceptual phenomenon "in which stimulation in one sensory or cognitive stream leads to associated experiences in a second, unstimulated stream" (Hubbard 2007). Neurocognitive models (Hubbard 2007) postulate the existence of an unusual communication between modality-specific brain areas, that is, the regions implicated in the processing of the inducing stimuli and the appearance of the synesthetic concurrents. In the literature, the nature of such unusual communication is explained by different models which are presented as either direct or indirect, and/or anatomical or functional (see the taxonomy of candidate neural mechanisms: Bargary and Mitchell 2008). Recently, on the basis of empirical evidence, an updated and integrative model, namely the cascaded cross-tuning model, has been proposed (Hubbard et al. 2011). However, the underlying neural mechanisms of synesthesia remain controversial, and new empirical information is needed to shed light on this question.

The first studies on the neuroanatomy of synesthesia assessed differences in connectivity (Rouw and Scholte 2007), cortical volume (Rouw and Scholte 2010; Weiss and Fink 2009) and both of the latter combined with the analysis of cortical thickness and cortical surface area (Jäncke et al. 2009). These studies, performed in grapheme-color synesthetes, used whole-brain and/or region of interest (ROI) analyses (Weiss and Fink 2009), and they paid special attention to two specific areas. The first area is the fusiform gyrus (FG) where hV4 lies. The color-selective area has shown differential activation in some grapheme-color functional experiments (Hubbard et al. 2005; Sperling et al. 2006; van Leeuwen et al. 2011; Specht and Laeng 2011), but not in others (Hupé et al. 2011; Rich et al. 2006; Rouw and Scholte 2007, 2010; Weiss et al. 2005). The other focus of interest for anatomical investigation has been the intraparietal sulcus (IPS), which is believed to mediate the hyperbinding of the inducer (e.g., a grapheme) and the concurrent (e.g., one specific color). The left IPS has shown differential activation in grapheme-color functional studies (van Leeuwen et al. 2011; Weiss et al. 2005), whereas stimulation of the right IPS with transcranial magnetic stimulation (TMS) resulted in the disruption of the Synesthetic Stroop Effect (Esterman et al. 2007; Muggleton et al. 2007; Rothen et al. 2010). Regarding neuroanatomical data, only 2 out of 4 studies of gray matter (GM) reported variations in the FG (Jäncke et al. 2009; Weiss and Fink 2009) and/ or the IPS (Rouw and Scholte 2010; Weiss and Fink 2009) (for a summary of published data on the neuroanatomy of developmental grapheme-color synesthesia, see Supplementary Material - Introduction: Table 1 ). In regard to white matter (WM) alterations, higher fractional anisotropy in the inferior temporal and left parietal areas has appeared only once (Rouw and Scholte 2007).

Nevertheless, these grapheme-color anatomical studies reported results not only in the FG and the IPS, but also in other areas (Supplementary Material - Introduction: Table 1). Additionally, a recent paper on grapheme-color anatomical peculiarities (gray and white matter volume) applied FWE correction for multiple comparisons and reported increased white matter volume in the retrosplenial cortex and the superior temporal sulcus only (Hupé et al. 2011). This can be due to the fact that the authors used a different method of analysis. Different analyses of white matter (FA vs. volume) provide different information about its structure (highly directional diffusion vs. higher density of connectivity fibers, respectively). Thus, both white matter volume results (Hupé et al. 2011) and FA data (Jäncke et al. 2009; Rouw and Scholte 2007) are informative in synesthesia research, and the combination of both (Hänggi et al. 2008) increases the detection power of anatomical studies (Gazdzinski et al. 2010). Moreover, another type of data analysis, based on connectivity matrices derived from region-wise cortical thickness correlations, has demonstrated widespread connectivity alterations in graphemecolor synesthetes (Hänggi et al. 2011).

Given this new empirical evidence, a whole-brain approach is still necessary to investigate the neuroanatomical basis of grapheme-color synesthesia. Firstly, because empirical results are not consistent enough to define the FG and the IPS as the only relevant areas of the synesthetic brain. Secondly, because these regions are defined over models that focus attention mainly on the modality-specific and top-down aspects of synesthesia, they do not take into account either subcortical contributions or other components of the experience, even though they are both justified and complementary lines of research.

The emotional component of synesthesia

Emotion is one of the components of synesthesia (Cytowic 2002) whose importance becomes more evident when the phenomenon is analyzed as a whole. This idea is supported by (a) the early associative theories which rely on the usefulness and pleasantness of the experience (Calkins 1895) (b) the claims about the emotional component of synesthesia (Cytowic and Eagleman 2009) (c) the idea that some types of synesthesia are emotionally mediated (Ward 2004) (d) the emotional displeasure provoked by incongruently 
colored graphemes (Ramachandran and Hubbard 2001b) (e) the behavioral evidence about synesthetically elicited affective reactions in grapheme-color synesthetes (Callejas et al. 2007) (f) the hypotheses about the hyperconnectivity between limbic and extra-striate regions (Ramachandran and Hubbard 2001a) (g) the neuroimaging evidence of unusual activity in the retrosplenial cortex (Nunn et al. 2002; Weiss et al. 2001) and the insula (Niccolai et al. 2012; Sperling et al. 2006; Specht and Laeng 2011) (h) the neuroanatomical evidence of variations in the cingulate gyrus, orbitofrontal cortex, insula (Jäncke et al. 2009) and the retrosplenial cortex (Hupé et al. 2011) and, last, but not least, (i) the synesthetes' accounts of their own experience. Despite the relevance of these claims, at present emotion is still not fully integrated in explanatory neurocognitive models.

This paper aims to disentangle the neural basis of the emotional component of grapheme-color synesthesia. Since the emotional processing recruits several neuronal circuits, the whole brain is analyzed. As described above, several cortical areas related to emotion have shown anatomical (Hupé et al. 2011; Jäncke et al. 2009) and functional (Nunn et al. 2002; Sperling et al. 2006; Specht and Laeng 2011; Weiss et al. 2001) peculiarities previously. Nevertheless, subcortical contributions are also relevant to complete the neuroemotional landscape, as we explain below.

\section{Subcortical contributions to developmental synesthesia}

The subcortical contribution to developmental synesthesia is still uncertain. However, empirical evidence regarding this matter can be found in a previous anatomical study. This was carried out on a multiple synesthete that exhibited interval-taste and tone color synesthesia. The authors reported increased gray matter volume in the synesthete's bilateral thalamus and the right nucleus accumbens (Hänggi et al. 2008). There also exist theoretical reasons to pay attention to subcortical structures. Luria suggested that an overactivation of subcortical structures together with a decreased cortical activity could result in synesthetic experiences (Luria 2006). In the same vein, more recently, the oscillatory resonance supervenience model (ORS) (Sidoroff-Dorso 2012), following the neurodynamical perspective (Thompson and Varela 2001), postulates the existence of a large-scale coherent oscillatory network that includes deep brain regions and can explain several dimensions of the synesthetic experience. Empirical evidence from acquired synesthesia supports this subcortical contribution hypothesis. Naumer and van den Bosch (2009) analyzed a paper that investigated synesthetic experiences after thalamic lesion (Beauchamp and Ro 2008) and remarked that alterations in thalamocortical projections contribute to the appearance of unusual multisensory integration phenomena (i.e., synesthesia). Furthermore, a recent publication
(Fornazzari et al. 2011) has reported a case of acquired synesthesia after thalamic stroke, including not only sensory manifestations (sound-tactile, sound-color, graphemegustatory) but also concept-triggered modalities. Moreover, a recent functional experiment about developmental grapheme-color synesthesia has shown unusual bilateral thalamic activation (Specht and Laeng 2011). Interestingly, recent evidence suggests that the thalamus and the basal ganglia may play a role in emotion (Carretie et al. 2009; Lane et al. 1997; Zeki and Romaya 2008). Therefore, the anatomy of subcortical structures is relevant to wholly understand the emotional component of synesthesia.

In this context, this study has investigated the grapheme-color synesthetic brain. To achieve this aim, 3T MRI whole-brain analyses were performed. 3D-T1-weighted and diffusion tensor images (DTI) were acquired from 8 grapheme-color synesthetes and 6 matched controls. The diffeomorphic anatomical registration through exponentiated lie algebra (DARTEL) method, which allows for more advanced coregistration (Ashburner 2007), provided a reliable definition of intergroup variability. Different anatomical indexes, including gray and white matter volume and fractional anisotropy (FA) analyses, were measured to draw a complete picture of the synesthetic brain. Subsequently, whole-brain results (including those found in deep brain structures) are discussed to better understand their contribution to the emotional component emphasized above.

\section{Methods}

\section{Participants}

Eight synesthetes and six controls matched for age (synesthetes' mean age $=24.88 \pm 3.44$; controls' mean age $=25.17 \pm 4.8$ ), sex (synesthetes: 7 women and 1 man; controls: 5 women and 1 men), handedness and education participated in this study. They had all completed the Synesthesia Battery grapheme-color task (Eagleman et al. 2007). Those who got punctuations between 0 and 1 were classified as synesthetes and only those who got punctuation well above 2 were selected as controls, in order to verify that there were no synesthetic experiences at all nor consistency. All control participants affirmed that they never experienced any of the synesthesias described in the battery. The participants had neither history of neurological, neurophysiological nor psychiatric disease and none of them reported drug abuse. They all had normal or corrected-to-normal visual acuity. Subjects were informed about the purpose of the investigation before the experimental session and signed a consent form according to the Declaration of Helsinki (for a detailed description of the sample, see Supplementary Material - Methods - Participants). 
Magnetic resonance imaging (MRI) data acquisition

All images were acquired on a $3.0 \mathrm{~T}$ Signa HDx scanner (GE Healthcare, Waukesha, WI). A 3D-T1-weighted gradient echo volume and a diffusion-weighted volume were acquired for each subject. The 3D-T1-weighted acquisitions parameters were as follows: $\mathrm{TE}=3.2 \mathrm{~ms}$, $\mathrm{TR}=7.2 \mathrm{~ms}$, TI $=750 \mathrm{~ms}$, flip-angle $=12^{\circ}$, slice thickness $=1 \mathrm{~mm}$, with no gap between slices, acquisition voxel size $=0.83 \times 0.83 \times 1 \mathrm{~mm}, \mathrm{FoV}=240 \mathrm{~mm}$ and sagittal acquisition with 154 slices. The diffusion-weighted image protocol acquisition consisted of one image without diffusion gradients $\left(b=0 \mathrm{~s} / \mathrm{mm}^{2}\right)$ followed by 15 images measured with 15 directions ( $b=1,000 \mathrm{~s} / \mathrm{mm}^{2}$ ) isotropically distributed in space. Additional parameters of the acquisition were as follows: $\mathrm{TE}=90 \mathrm{~ms}$, TR $=9835 \mathrm{~ms}$, flip-angle $=90^{\circ}$, slice thickness $=3 \mathrm{~mm}$, gap between slices $=0.3 \mathrm{~mm}$, acquisition voxel size $=1.875 \times 1.875 \times 3 \mathrm{~mm}, \mathrm{FoV}=240 \mathrm{~mm}$ and axial acquisition with 36 slices.

\section{D-T1-weighted image pre-processing}

The anatomical 3D data were processed using SPM8 (Statistical Parametric Mapping, Wellcome Department of Cognitive Neurology, University College of London, UK). First of all, the MR images were segmented into GM, WM and cerebrospinal fluid (CSF) using the standard unified segmentation model (Ashburner and Friston 2005). Second, the SPM8 toolbox named DARTEL (Ashburner 2007) was used to generate a template for GM and WM. Only the control group was used to create this template, given that control-created templates can provide greater detection accuracy than those that include all subjects, even when this control group is small (Shen et al. 2007). GM and WM segmented images were registered and normalized to the template using diffeomorphisms (Singh et al. 2012). This method improves the image registration, which results in a better localization and increased sensitivity in analysis ( $\mathrm{Li}$ et al. 2010). Modulation was applied to correct voxel signal intensity for volume displacement during normalization (Good et al. 2001). Given our interest in subcortical regions and our nonparametric approach (Nichols and Holmes 2002), the images were smoothed with a narrow $4 \times 4 \times 4 \mathrm{~mm}$ FWHM Gaussian kernel to improve the signal-to-noise ratio and to avoid partial volume effects but preserving the morphological details. These registered, normalized, modulated and smoothed images were used in the statistical analysis.

\section{Diffusion tensor image pre-processing}

An analysis of diffusion tensor images was performed to investigate possible differences in fractional anisotropy. A
FA map was calculated using Functool software (GE 4.3. Advantage Windows WS), correcting the images for head motion and eddy current artifacts. First, the $b=0$ images were registered to the T2 template, included in the SPM8 package. The spatial transformation was applied to the FA maps. After that, the $b=0$ images of the control group were normalized to the T2 template. The spatial transformation was again applied to the FA maps. These normalized FA maps were smoothed with a $4 \times 4 \times 4 \mathrm{~mm}$ FWHM Gaussian kernel to minimize partial volume effect and averaged to create a FA template from the control group. Finally, every initially registered FA map was normalized to the FA template and smoothed with a $4 \times 4 \times 4 \mathrm{~mm}$ FWHM Gaussian kernel. This normalized and smoothed images were used to perform the voxel-based analysis (Kunimatsu et al. 2007).

\section{Statistical analysis}

Statistical analysis was performed using the Statistical nonParametric Mapping toolbox (SnPM: Nichols and Holmes 2002), which is more suitable for designs with low degrees of freedom available for variance estimation. It uses the general linear model (GLM) to construct the pseudo-t-statistics images. These images were used to study intergroup differences in each tissue (GM and WM), as well as in FA.

The statistical test used to compare the different groups was a 2-sample $t$ test applied to nonparametric analysis (Nichols and Holmes 2002). Age, gender and intracranial volume were introduced as confounding covariates for the analysis. Whole-brain analyses (synesthetes $>$ controls) were performed, with a threshold of $p_{\text {unc }}<.001$. In the case of subcortical data, the inverse contrast (controls $>$ synesthetes; $p_{\text {unc }}<.001$ ) was also analyzed, to test the relation between synesthesia acquired after lesion and developmental synesthesia. To explore the areas described by other authors (see Supplementary Material - Introduction: Table 1) and to minimize the risk of losing information due to individual differences in the synesthetes studied by different researchers (Rouw and Scholte 2010), a less strict threshold ( $p_{\text {unc }}<.01$ ) was applied. The statistical threshold was lowered (whole-brain analysis; $p_{\text {unc }}<.01$ ) in order to perform this comparison, and every coordinate reported in Table 1 (Supplementary Material - Introduction) was tested (distance criterion $<8 \mathrm{~mm}$ ). The final SnPM images were superimposed on a T1 template or a FA template with MRIcrogl software. Anatomical identification was performed via XjView 8 (http://www.alivelearn.net/xjview8/) and the anatomy toolbox by Eickhoff et al. (2005). The Oxford Thalamic Connectivity Probability Atlas was used for the identification of areas of the cortex where thalamic nucleus project (Johansen-Berg et al. 2005). In addition, for the labeling of anatomical results, the neuroanatomy atlases 
Table 1 Variations in the synesthetic brain (synesthetes > controls; $\left.p_{\text {unc }}<.001\right)$

\begin{tabular}{|c|c|c|c|c|c|c|c|}
\hline \multirow[t]{2}{*}{ Measurement } & \multirow[t]{2}{*}{ Region } & \multirow[t]{2}{*}{ Hemisphere } & \multirow{2}{*}{$\begin{array}{l}\text { Cluster size } \\
\text { (vx) }\end{array}$} & \multicolumn{3}{|c|}{ MNI coordinates } & \multirow[t]{2}{*}{$t$} \\
\hline & & & & $\mathrm{x}$ & $\mathrm{y}$ & $\mathrm{z}$ & \\
\hline \multirow{4}{*}{$\begin{array}{l}\text { Volume } \\
\text { (gray matter) }\end{array}$} & Parahippocampal gyrus & $\mathrm{L}$ & 8 & -18 & -2 & -26 & 3.62 \\
\hline & Putamen & $\mathrm{L}$ & 47 & -24 & 6 & 6 & 4.98 \\
\hline & Middle frontal gyrus & $\mathrm{L}$ & 27 & -24 & 38 & 28 & 4.12 \\
\hline & Postcentral gyrus & $\mathrm{R}$ & 8 & 40 & -30 & 66 & 3.52 \\
\hline $\begin{array}{l}\text { Volume } \\
\text { (white matter) }\end{array}$ & Temporal pole & $\mathrm{R}$ & 17 & 28 & 24 & -34 & 4.25 \\
\hline \multirow[t]{6}{*}{ FA } & Middle frontal subgyral & $\mathrm{L}$ & 85 & -36 & 18 & 32 & 5.11 \\
\hline & Inferior frontal gyrus & $\mathrm{L}$ & 5 & -50 & 14 & 16 & 3.54 \\
\hline & Anterior insula & $\mathrm{R}$ & 45 & 38 & 30 & 6 & 4.57 \\
\hline & Cuneus & $\mathrm{L}$ & 13 & -14 & -90 & 32 & 3.82 \\
\hline & Middle occipital gyrus & $\mathrm{L}$ & 12 & -38 & -98 & -4 & 3.51 \\
\hline & Cingulate gyrus & $\mathrm{L}$ & 5 & -10 & -6 & 42 & 3.15 \\
\hline
\end{tabular}

by Haines (2011) and Nolte and Angevine (2007) were consulted.

\section{Results}

Whole-brain analysis revealed anatomical peculiarities mainly within the left hemisphere of the synesthetic brain, though some results appeared also on the right hemisphere. Table 1 shows areas of the brain where synesthetes exhibit increased volume and higher FA values.

\section{Emotional areas}

Several regions where synesthetes present higher gray and/ or white matter volume and/or higher fractional anisotropy values are related to emotion (Fig. 1). Increased gray matter volume $\left(p_{\text {unc }}<.001\right)$ was observed in the synesthetes' left parahippocampal gyrus, when compared with the control group. In the case of white matter volume, variations appeared in the right temporal pole $\left(p_{\text {unc }}<.001\right)$. FA differences were observed in the white matter underlying the right anterior insula and the cingulate gyrus $\left(p_{\text {unc }}<.001\right)$.

\section{Subcortical areas}

Synesthetes showed increased gray matter volume in the left putamen $\left(p_{\text {unc }}<.001\right)$. Given the empirical evidence that describes the appearance of synesthetic experiences as a consequence of thalamic lesion, and to understand the relation between acquired and developmental synesthesia, the inverse contrast (controls $>$ synesthetes; $p_{\text {unc }}<.001$ ) was also analyzed. Using this threshold, a great cluster of thalamic results appeared. To understand the location of these thalamic variations, a stricter threshold was applied $\left(p_{\text {unc }}<.0001\right)$. Interestingly, controls still showed

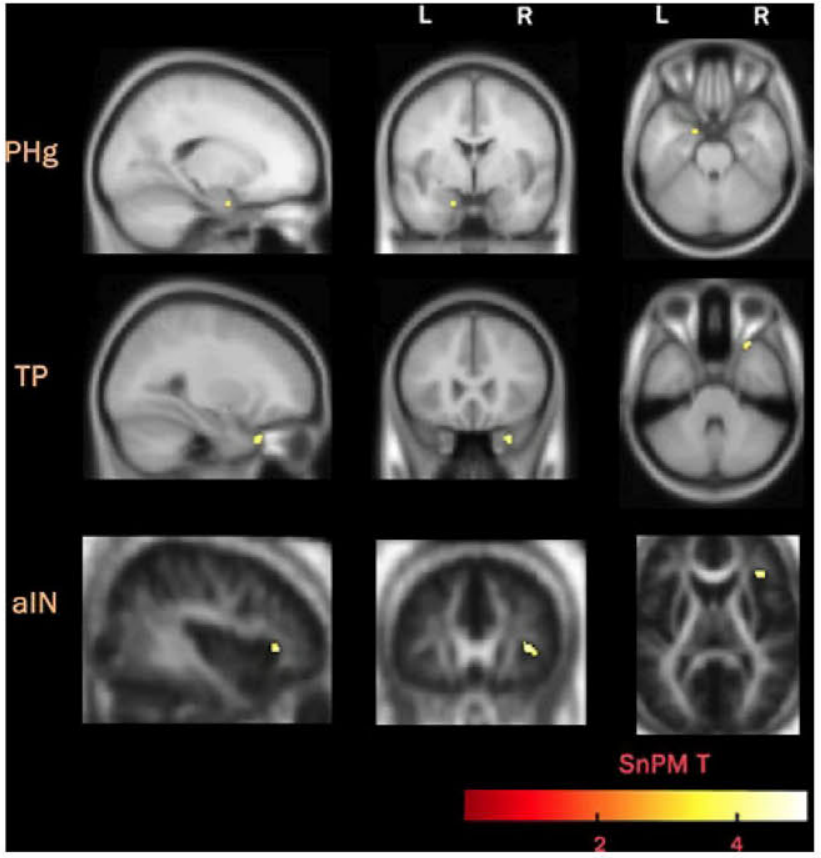

Fig. 1 Emotional areas where synesthetes exhibit increased volume and higher FA values than controls. First row increased gray matter volume in the left parahippocampal gyrus (PHg); Second row increased white matter volume in the right temporal pole (TP); Third row higher FA values in the right anterior insula (aIN)

significant increased white matter volume (Fig. 2a) and increased FA values (Fig. 2b) in the thalamus (Table 2).

The canonical regions of interest

The whole-brain analysis $\left(p_{\text {unc }}<.001\right)$ did not reveal any significant difference in the canonical regions of interest (i.e., FG and/or the IPS). To test the gray matter similarities between the participant grapheme-color synesthetes 


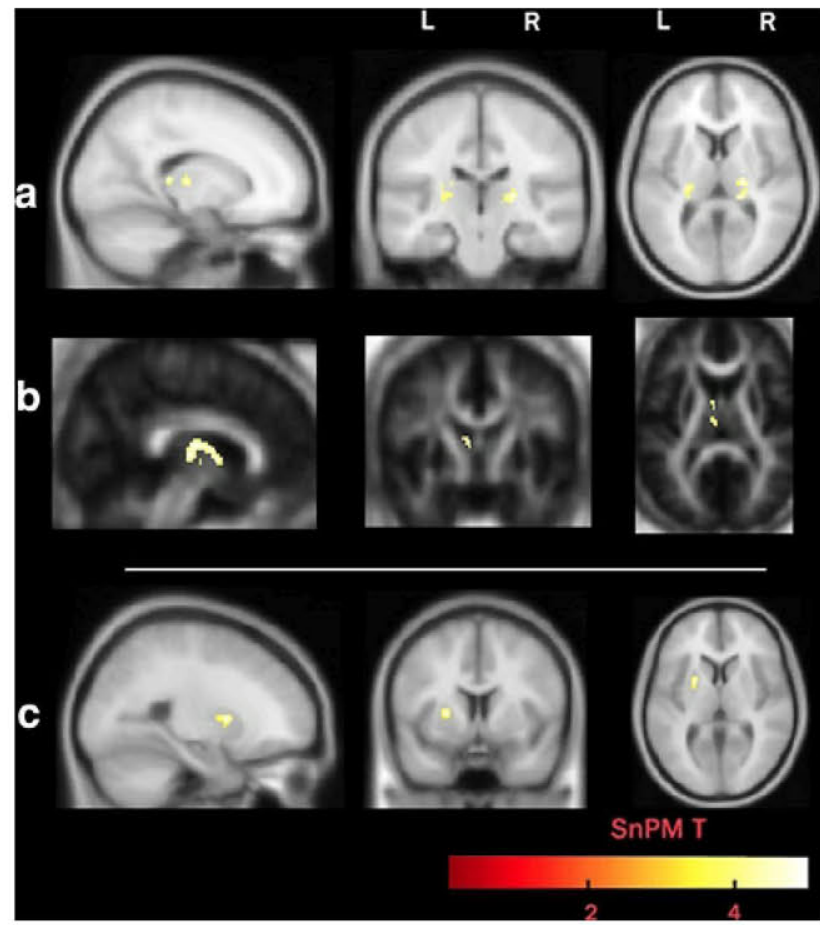

Fig. 2 Subcortical anatomical differences: a increased white matter volume in the left and right thalamus; controls > synesthetes $\left(p_{\text {unc }}<.0001\right)$ b increased fractional anisotropy in the left thalamus; controls $>$ synesthetes $\left(p_{\text {unc }}<.0001\right) \mathbf{c}$ increased gray matter volume in the left putamen; synesthetes $>$ controls $\left(p_{\text {unc }}<.001\right)$ in this study and previous studies, the areas reported in previous research (Jäncke et al. 2009; Rouw and Scholte 2010; Weiss and Fink 2009) were explored using a less strict threshold, as has been done to compare results from different studies previously (Jäncke et al. 2009). A new whole-brain analysis $\left(p_{\text {unc }}<.01\right)$ was performed, and every region reported in Table 1 (Supplementary Material - Introduction) was explored (distance criterion $<8 \mathrm{~mm}$ ). Significant gray matter differences $\left(p_{\text {unc }}<.01\right.$; synesthetes $>$ controls) were observed only in the vicinity of the region described by Weiss et al. (2009) as the left caudal IPS. The anatomy toolbox by Eickhoff et al. (2005) suggests that our significant cluster is located near the left intraparietal area (hIP3) (Supplementary Material -Results: Figure 1). The same procedure was followed to investigate white matter volume and FA data. No differences in grapheme and/or color FG areas or posterior IPS regions were found.

\section{Discussion}

Synesthesia is a conscious perceptual phenomenon with a neurocognitive basis. Functional and anatomical studies have provided evidence on the perceptual reality of the experience, showing that relevant areas of the brain for

Table 2 Thalamic anatomical differences (controls $>$ synesthetes; $p_{\text {unc }}<.0001$ )

\begin{tabular}{|c|c|c|c|c|c|c|c|c|}
\hline \multirow[t]{2}{*}{ Measurement } & \multirow[t]{2}{*}{ Region } & \multirow{2}{*}{$\begin{array}{l}\text { Highest probability } \\
\text { Projection area }^{\mathrm{a}}\end{array}$} & \multirow[t]{2}{*}{ Hemisphere } & \multirow{2}{*}{$\begin{array}{l}\text { Cluster Size } \\
(\mathrm{vx})\end{array}$} & \multicolumn{3}{|c|}{ MNI coordinates } & \multirow[t]{2}{*}{$t$} \\
\hline & & & & & $\mathrm{x}$ & $\mathrm{y}$ & $\mathrm{z}$ & \\
\hline \multirow{5}{*}{$\begin{array}{l}\text { Volume (white } \\
\text { matter) }\end{array}$} & Thalamus: & & & & & & & \\
\hline & $\begin{array}{r}\text { Ventral posterior } \\
\text { lateral Nucleus }\end{array}$ & Posterior parietal Cortex & $\mathrm{R}$ & 20 & 20 & -22 & 8 & 4.52 \\
\hline & $\begin{array}{l}\text { Ventral posterior } \\
\text { medial Nucleus }\end{array}$ & Unclear projection $^{\mathrm{b}}$ & $\mathrm{R}$ & 7 & 16 & -20 & 8 & 4.07 \\
\hline & Pulvinar & Temporal cortex & $\mathrm{R}$ & 33 & 18 & -32 & 8 & 4.66 \\
\hline & & Posterior parietal cortex & $\mathrm{L}$ & 17 & -20 & -24 & 8 & 4.148 \\
\hline \multirow[t]{5}{*}{ FA } & Thalamus: & & & & & & & \\
\hline & $\begin{array}{l}\text { Medial dorsal } \\
\text { nucleus }\end{array}$ & Temporal cortex & $\mathrm{L}$ & 31 & -6 & -12 & 12 & 4.18 \\
\hline & & Pre-frontal cortex & & & -6 & -14 & 6 & 4.85 \\
\hline & & Temporal cortex & & & -4 & -12 & 12 & 4.76 \\
\hline & $\begin{array}{l}\text { Ventral anterior } \\
\text { nucleus }\end{array}$ & Temporal cortex & $\mathrm{L}$ & 27 & -6 & -2 & 8 & 4.19 \\
\hline
\end{tabular}

In columns: measurement (volume or FA data), location (derived from XjView 8 software: http://www.alivelearn.net/xjview8/), area of the cortex where the described thalamic locations project with high probability, brain hemisphere (left or right), cluster size in voxels, MNI coordinates and $t$ value

${ }^{\text {a }}$ From Oxford Thalamic Connectivity Probability Atlas

${ }^{\mathrm{b}}$ Highest probability projection area under $50 \%$ 
the processing of the inducer and the concurrent, as well as higher order regions, could take part in its generation. Given the existence of empirical and phenomenological evidence about the emotional component of synesthesia, this study has investigated both cortical and subcortical structures in order to disentangle the underlying neural network which supports it.

\section{Synesthesia and emotion}

As described above, there exists theoretical, empirical and phenomenological reason to look for the neuroemotional basis of synesthesia. Several areas reported in studies of synesthesia have been also described as "the most frequent regional activations reported in 51 emotion-related experiments from 25 publications" (Maddock 1999). For example, the cingulate gyrus has appeared repeatedly in functional (Nunn et al. 2002; Weiss et al. 2001) and anatomical (Hupé et al. 2011) research. The finding of increases of white matter volume in the retrosplenial cortex (RSC) bilaterally, and the absence of significant results in color areas, led Hupé et al. (2011) to reconsider the nature of the synesthetic color. They hypothesized that this area contributes to bind visual attributes to emotion and memories to create meaningful connections. Having in mind the role of the RSC in the processing of emotionally salient stimuli (Maddock 1999), they suggest that meaning itself, not color, is the relevant feature of synesthetic associations and redefined these associations as "memorized (arbitrary and idiosyncratic) associations loaded with emotional content" (Hupé et al. 2011).

The anatomical results presented here provide new insight into this question. Our finding in the cingulate cortex is more rostrally located (anterior cingulate gyrus (ACG)/Brodmann's Area 24). Interestingly, this area participates in the integration of attentional and emotional networks (Fichtenholtz et al. 2004; Lane et al. 1997; Yamasaki et al. 2002) and is connected to the middle frontal gyrus (MdFG) (Koski and Paus 2000). The grapheme-color synesthetes who took part in our study show variations in this area (MdFG), both increases in gray matter volume (replicating the finding of Rouw and Scholte 2010) and higher FA values. This region has also shown unusual activity during synesthetic tasks previously (Rouw and Scholte 2007; Specht and Laeng 2011). Other authors have interpreted the unusual activation of MdFG as a component of the attentional and/or perceptual networks that could give rise to synesthetic experiences (Specht and Laeng 2011). Since this area also participates in top-down emotional processing (Ochsner et al. 2009), and given its connections to the ACG, variations in both areas are interpreted here as part of the emotional network that could be at the root of synesthetic experiences.
Other variations reported here support the existence of this peculiar emotional network. The insula has appeared repeatedly in functional (Sperling et al. 2006; Specht and Laeng 2011) and anatomical (Jäncke et al. 2009) grapheme-color research, and its anterior part has shown higher FA values in our sample. This area participates in taste and smell but also in sensoriomotor and cognitive tasks and in social-emotional processing (Kurth et al. 2010). These same authors found that its anterior part works as a functional integrator for different functional systems, so we suggest that it could be a central area in the integration processes that contributes to the appearance of synesthetic experiences in general, not only in the grapheme-color modality. The pattern of connectivity of this area also supports this hypothesis. The anterior insula projects to the pregenual ACG (Taylor et al. 2009), and it is strongly connected to the amygdala complex (Mufson et al. 1981). The present study has also reported significant results in the vicinity of the amygdala complex. Specifically, our sample shows increased gray and white matter volume in the parahippocampal gyrus and the temporal pole, respectively. The role of the temporal pole (TP) in the emotional processing of visual, olfactory and auditory stimuli and its proposed general function in the coupling of emotional responses to highly processed perceptual inputs (Olson et al. 2007) make plausible its contribution to the cognitive-emotional network that could underlie synesthetic integration.

Though all these regions could be implicated in other cognitive processes (i.e., attention, memory and conscious perception), there is no reason to discard their emotional contribution to grapheme-color synesthesia. The facts that they are interconnected and belong to emotional networks, argue for their relevance in the generation of synesthetic experiences. Nevertheless, further functional research is needed to discern their specific contribution to synesthesia.

Synesthesia and subcortical gray nuclei

As described above, theoretical models and empirical evidence support the idea that thalamic variations could account for unusual activity in cortical areas (i.e., synesthetic experiences). Thalamic alterations have not been reported in any anatomical study of developmental grapheme-color synesthesia previously. Thus, for the first time, this work provides evidence of decreased white matter volume and FA around the thalamus in grapheme-color synesthetes. This variation could be interpreted as a lower degree of connectivity between the thalamus and the cortex that leads to unusual connectivity patterns (e.g., inter/intra sensory pathways) which crystallize in different sensory manifestations (e.g., grapheme-color synesthesia). This finding is consistent with the described empirical evidence about thalamic lesions leading to synesthesia (Beauchamp and Ro 
2008; Fornazzari et al. 2011; Naumer and van den Bosch 2009), bearing in mind that developmental synesthesia is not usually associated with sensory deficit. In our sample, lower values of white matter volume and FA were found in several thalamic nuclei. The Oxford Thalamic Connectivity Probability Atlas reveals that these nuclei where decreased white matter volume and lower FA values appear project mainly to the temporal and posterior parietal cortex. Only one study of synesthesia (in a multiple synesthete nonincluding grapheme-color synesthesia) reported anatomical variations in the thalamic nuclei (Hänggi et al. 2008). Curiously, their results (increased gray matter volume in the thalamus of synesthetes) appear in nuclei that project to these same locations (temporal and posterior parietal cortex). Therefore, empirical data suggest that subcortical structures participate in synesthesia, but functional experiments and further anatomical research, which analyze thalamic variations and its projections to cortical areas, are needed to understand the role that subcortical structures play in synesthesia.

Subcortical variations appear also in the basal ganglia. Increased gray matter volume was found in the left putamen in the synesthetic group, a result that also fits with an emotional explanation. As mentioned above, putamen is one of the areas of the brain which appears repeatedly in emotion-related experiments (Maddock 1999). Furthermore, this basal ganglion has been described as part of the hate circuit (Zeki and Romaya 2008), and it contributes to the pattern of activation that arises when studying the neural correlates of romantic love (Bartels and Zeki 2000).

\section{The canonical regions of interest}

As described in the Introduction section, there has been a tendency to look for anatomical variations in specific locations of the synesthetic brain, namely the FG and the IPS. The results presented here show no variations in the grapheme and color processing areas of the FG. Therefore, including the present work, 3 out of 5 structural analyses of gray matter (Hupé et al. 2011; Rouw and Scholte 2010) and 3 out of 4 structural studies on white matter (Hupé et al. 2011; Jäncke et al. 2009) failed to find alterations in this area in grapheme-color synesthetes. Given these results, and the inconsistent data of differential activation of V4 described in the Introduction section, there is not enough evidence to conclude that anatomical variations in modality-specific areas of the cortex are necessary to understand grapheme-color synesthesia. Regarding parietal areas, our synesthetic participants showed increased cortical volume in the left IPS replicating previous anatomical (Rouw and Scholte 2007, 2010; Weiss and Fink 2009) and functional results (van Leeuwen et al. 2011; Weiss et al. 2005). Parietal variations were also described by Jäncke et al. (2009) although in a different location (right precuneus). Thus, 5 out of 6 neuroanatomical studies on developmental grapheme-color synesthesia report parietal results. Given this state of affairs and TMS evidence (Esterman et al. 2007; Muggleton et al. 2007; Rothen et al. 2010), parietal results are consistent enough to define this region (IPS) as a crucial area in grapheme-color synesthesia.

Though neuroanatomical variations seem to be consistently present in different samples of synesthetes, the debate on how these alterations appear remains open. Some authors suggest that the existence of a structural basis is necessary to understand the functional dynamics of synesthesia; this anatomical extraconnectivity may be the result of a heritable defective pruning (Ramachandran and Hubbard 2001a). Indeed, this incomplete apoptosis could contribute to the maintenance of intrinsic intersensory pathways that exists in early childhood (Spector and Maurer 2009). An alternative explanation is that these unusual pathways are the consequence of synesthetic experiences (Jäncke et al. 2009; Weiss and Fink 2009). Given that gray matter volume changes in the human adults' brain as a result of short periods of training have been demonstrated (Kwok et al. 2011), and that brain dynamics are claimed to promote neuroanatomical self-organization (Rubinov et al. 2009), the interpretation of synesthetic anatomical peculiarities as a result of repetitive intersensory coupling is not implausible. In this case, functional imbalance would be responsible for structural variations. When considered from a dynamical perspective, this is not an all or none problem. The existence of a structurally different emotional network could elicit a functional imbalance that crystallizes in functional variations in different sensory systems, eliciting specific types of synesthesia. Indeed, the information obtained from the use of different structural measures of the brain points in this direction. The existence of alterations in deep brain structures, both in gray matter (e.g., increased gray matter volume in the putamen) and white matter (e.g., decreased white matter volume and FA values in the thalamus) suggests that synesthesia does not depend only on cortical networks. This, together with (a) macrostructural variations (i.e., increased volume) in the parahippocampal gyrus (gray matter) and the temporal pole (white matter), which complement each other, (b) microstructural white matter findings (i. e., higher FA values), which reveal higher directionality of the fibers in the insula, anterior cingulate and middle frontal areas and (c) the absence of variations in modality-specific areas, reinforce the crucial role of other networks (e.g., emotional networks) in graphemecolor synesthesia.

It is certainly difficult to determine the causes for the existence of an unusually interconnected emotional network that we could call Emotional Binding System. However, this emotional hypothesis is consistent with the 
neurochemical one. The psychopharmacology of synesthesia remains unknown, but the receptor $\mathrm{S} 2 \mathrm{a}$, and thus, the serotonin system has been claimed to be a good candidate to mediate synesthetic experiences (Brang and Ramachandran 2008). Serotonin is implicated in early stages of the nervous system development as well as in adult plasticity (Whitaker-Azmitia 2010), and therefore, physiological differences in this neurotransmitter system could contribute to the appearance of structural peculiarities in the synesthetic brain, including the neural circuits and brain structures involved in emotions. Future large-scale research assessing the emotional characteristics of synesthetes (not only in relation to synesthesia but to perception in general) will help clarify the relevance of the emotional component in the generation of unusual functioning of the sensory systems.

This study is no exception in having limitations. The first limitation has to do with the lack of a quantitative measure of the strength and valence of the emotional characteristics of our participants' synesthesia. Though the emotional strength of grapheme-color synesthesia has been described in the literature previously (Callejas et al. 2007), behavioral measures of the emotional component of synesthesia are still necessary to interpret these anatomical data and fully account for the emotional binding hypothesis. The second limitation concerns the sample size. Though this limitation has been minimized with the nonparametric statistical approach, it affects the extent to which the findings can be generalized beyond the cases studied. Nonetheless, results from small synesthetic samples (e.g., Hubbard et al. 2005 $=6$ synesthetes; Brang and Ramachandran $2008=4$ synesthetes) constitute the seed to explore new dimensions of synesthesia and thus go further in our understanding of the phenomenon. The third limitation is that none of our results survived correction for multiple comparisons, which is a common tendency in synesthesia studies (see Supplementary Material - Introduction: Table 1). In our case, both the use of an alternative statistical approach (nonparametric tests, given the size of our sample) and the finding of significant results even with a small spatial filter $(4 \mathrm{~mm})$, that is less likely to give significant results (Jones et al. 2005), account for the reliability of the reported data. Despite these limitations and other possible artifacts arising from methodological constraints, the combination of VBM and DTI has provided accurate information about the nature of gray and white matter in the brain of synesthetes and has revealed structural data that are relevant to find a bridge between developmental and acquired synesthesia.

In summary, our results are consistent with previous anatomical research in one of the canonical regions of interest (IPS) and, as we expected, reveal the existence of variations in emotion-related areas of the synesthetic brain. Additionally, increased gray matter volume in the putamen and lower FA and white matter volume in the thalamus were observed. These findings favor the idea that, at least in our sample of associator synesthetes, the structural basis is not modality specific but distributed both at the cortical and subcortical level. The nonparametric statistical approach allows restricted inference about other populations. However, the data provided here will be useful for a better definition of the synesthetic experience, as it provides new insight into the neural basis of the still insufficiently explored but critical emotional component of synesthesia.

Acknowledgments We thank the participants and the technical staff at the 3T scanner facility of Fundación Centro de Investigación de Enfermedades Neurológicas for their indispensable collaboration in this study. Financial support was provided by Universidad Complutense de Madrid, Fundación Centro de Investigación de Enfermedades Neurológicas and Ministerio de Ciencia e Innovación PSI2009-14415-C03-03. The authors declare that they have no competing interests. This work conforms to Standard 8 of the American Psychological Association's Ethical Principles of Psychologist and Code of Conduct.

\section{References}

Ashburner J (2007) A fast diffeomorphic image registration algorithm. Neuroimage 38(1):95-113. doi:10.1016/j.neuroim age.2007.07.007

Ashburner J, Friston KJ (2005) Unified segmentation. Neuroimage 26(3):839-851. doi:10.1016/j.neuroimage.2005.02.018

Bargary G, Mitchell KJ (2008) Synaesthesia and cortical connectivity. Trends Neurosci 31(7):335-342. doi:10.1016/j.tins.2008.03.007

Bartels A, Zeki S (2000) The neural basis of romantic love. NeuroReport 11(17):3829-3834. doi:10.1097/00001756200011270-00046

Beauchamp MS, Ro T (2008) Neural substrates of sound-touch synesthesia after a thalamic lesion. J Neurosci 28(50):13696-13702. doi:10.1523/jneurosci.3872-08.2008

Brang D, Ramachandran VS (2008) Psychopharmacology of synesthesia; the role of serotonin S2a receptor activation. Med Hypotheses 70(4):903-904

Calkins MW (1895) Synaesthesis (minor studies from Wellesley College). Am J Psychol 7(1):90-107

Callejas A, Acosta A, Lupianez J (2007) Green love is ugly: emotions elicited by synesthetic grapheme-color perceptions. Brain Res 1127:99-107. doi:10.1016/j.brainres.2006.10.013

Carretie L, Rios M, de la Gandara BS, Tapia M, Albert J, LopezMartin S, Alvarez-Linera J (2009) The striatum beyond reward: caudate responds intensely to unpleasant pictures. Neuroscience 164(4):1615-1622

Cytowic RE (2002) Touching tastes, seeing smells and shaking up brain science. Cerebrum 4(3):7-26

Cytowic ER, Eagleman DM (2009) Wednesday is indigo blue: discovering the brain of synesthesia. MIT Press, Cambridge

Eagleman DM, Kagan AD, Nelson SS, Sagaram D, Sarma AK (2007) A standardized test battery for the study of synesthesia. J Neurosci Meth 159(1):139-145. doi:10.1016/j.jneumeth.2006.07.012

Eickhoff S, Stephan K, Mohlberg H, Grefkes C, Fink G, Amunts K, Zilles K (2005) A new SPM toolbox for combining probabilistic cytoarchitectonic maps and functional imaging data. Neuroimage 25(4):1325-1335. doi:10.1016/j.neuroimage.2004.12.034 
Esterman M, Verstynen T, Robertson LC (2007) Attenuating illusory binding with TMS of the right parietal cortex. Neuroimage 35(3):1247-1255. doi:10.1016/j.neuroimage.2006.10.039

Fichtenholtz HM, Dean HL, Dillon DG, Yamasaki H, McCarthy G, LaBar KS (2004) Emotion-attention network interactions during a visual oddball task. Brain Res Cogn Brain Res 20(1):67-80. doi:10.1016/j.cogbrainres.2004.01.006

Fornazzari L, Fischer CE, Ringer L, Schweizer TA (2011) 'Blue is music to my ears': multimodal synesthesias after a thalamic stroke. Neurocase. doi:10.1080/13554794.2011.608362

Gazdzinski S, Durazzo TC, Mon A, Yeh PH, Meyerhoff DJ (2010) Cerebral white matter recovery in abstinent alcoholics-a multimodality magnetic resonance study. Brain 133(Pt 4):1043-1053. doi:10.1093/brain/awp343

Good CD, Johnsrude IS, Ashburner J, Henson RN, Friston KJ, Frackowiak RS (2001) A voxel-based morphometric study of ageing in 465 normal adult human brains. NeuroImage 14(1 Pt 1):21-36. doi:10.1006/nimg.2001.0786

Haines D (2011) Neuroanatomy: an atlas of structures, sections and systems. 8th edn, Wolters Kluwer (Health)/Lippincott Williams \& Wilkins, Philadelphia

Hänggi J, Beeli G, Oechslin MS, Jäncke L (2008) The multiple synaesthete E.S.: neuroanatomical basis of interval-taste and tone-colour synaesthesia. Neuroimage 43(2):192-203. doi: 10.1016/j.neuroimage.2008.07.018

Hänggi J, Wotruba D, Jancke L (2011) Globally altered structural brain network topology in grapheme-color synesthesia. J Neurosci 31(15):5816-5828. doi:10.1523/JNEUROSCI.0964-10.2011

Hubbard EM (2007) Neurophysiology of synesthesia. Curr Psychiatry Rep 9(3):193-199

Hubbard EM, Arman AC, Ramachandran VS, Boynton GM (2005) Individual differences among grapheme-color synesthetes: brainbehavior correlations. Neuron 45(6):975-985. doi:10.1016/j. neuron.2005.02.008

Hubbard EM, Brang D, Ramachandran VS (2011) The crossactivation theory at 10. J Neuropsychol 5(2):152-177. doi:10.1111/j.1748-6653.2011.02014.x

Hupé JM, Bordier C, Dojat M (2011) The neural bases of graphemecolor synesthesia are not localized in real color-sensitive areas. Cereb Cortex 2(7):1622-1633. doi:10.1093/cercor/bhr236

Jäncke L, Beeli G, Eulig C, Hänggi J (2009) The neuroanatomy of grapheme-color synesthesia. Eur J Neurosci 29(6):1287-1293. doi:10.1111/j.1460-9568.2009.06673.x

Johansen-Berg H, Behrens TE, Sillery E, Ciccarelli O, Thompson AJ, Smith SM, Matthews PM (2005) Functional-anatomical validation and individual variation of diffusion tractography-based segmentation of the human thalamus. Cereb Cortex 15(1):31-39. doi:10.1093/cercor/bhh105bhh105

Jones DK, Symms MR, Cercignani M, Howard RJ (2005) The effect of filter size on VBM analyses of DT-MRI data. NeuroImage 26(2):546-554. doi:S1053-8119(05)00095-9

Koski L, Paus T (2000) Functional connectivity of the anterior cingulate cortex within the human frontal lobe: a brain-mapping meta-analysis. Exp Brain Res 133(1):55-65. doi:10.1007/ s002210000400

Kunimatsu A, Itoh D, Nakata Y, Kunimatsu N, Aoki S, Masutani Y, Abe O, Yoshida M, Minami M, Ohtomo K (2007) Utilization of diffusion tensor tractography in combination with spatial normalization to assess involvement of the corticospinal tract in capsular/pericapsular stroke: feasibility and clinical implications. J Magn Reson Imaging 26(6):1399-1404. doi:10.1002/j mri. 20945

Kurth F, Zilles K, Fox PT, Laird AR, Eickhoff SB (2010) A link between the systems: functional differentiation and integration within the human insula revealed by meta-analysis. Brain Struct Funct 214(5-6):519-534. doi:10.1007/s00429-010-0255-Z
Kwok V, Niu Z, Kay P, Zhou K, Mo L, Jin Z, So KF, Tan LH (2011) Learning new color names produces rapid increase in grey matter in the intact adult human cortex. Proc Natl Acad Sci U S A 108(16):6686-6688. doi:10.1073/pnas.1103217108

Lane RD, Reiman EM, Bradley MM, Lang PJ, Ahern GL, Davidson RJ, Schwartz GE (1997) Neuroanatomical correlates of pleasant and unpleasant emotion. Neuropsychologia 35(11): $1437-1444$

Li W, He H, Lu J et al (2010) Evaluation of multiple voxel-based morphometry approaches and applications in the analysis of white matter changes in temporal lobe epilepsy. In: Proceedings of the 5 th international workshop on medical imaging and augmented reality, Bejiing, Sept 19-20, 2010, http://www.miar.org/2010/ files/MIAR2010_3-2.pdf

Luria AR (2006) Lectures in general psychology. Piter Publishing House, Saint-Petersburg, pp 106-108 Reprinted from 1970-75 lectures

Maddock RJ (1999) The retrosplenial cortex and emotion: new insights from functional neuroimaging of the human brain. Trends Neurosci 22(7):310-316

Mufson EJ, Mesulam MM, Pandya DN (1981) Insular interconnections with the amygdala in the rhesus monkey. Neuroscience 6(7):1231-1248

Muggleton N, Tsakanikos E, Walsh V, Ward J (2007) Disruption of synaesthesia following TMS of the right posterior parietal cortex. Neuropsychologia 45(7):1582-1585. doi:10.1016/j.neuropsychol ogia.2006.11.021

Naumer MJ, van den Bosch JJ (2009) Touching sounds: thalamocortical plasticity and the neural basis of multisensory integration. J Neurophysiol 102(1):7-8. doi:10.1152/jn.00209.2009

Niccolai V, van Leeuwen TM, Blakemore C, Stoerig P (2012) Synaesthetic perception of colour and visual space in a blind subject: an fMRI case study. Conscious Cogn 21(2):889-899. doi: $10.1016 / j . \operatorname{con} \operatorname{cog} .2012 .03 .010$

Nichols TE, Holmes AP (2002) Nonparametric permutation tests for functional neuroimaging: a primer with examples. Hum Brain Mapp 15(1):1-25. doi:10.1002/hbm. 1058

Nolte J, Angevine J (2007) The human brain in photographs and diagrams, 3rd edn. Mosby Elsevier, Philadelphia

Nunn JA, Gregory LJ, Brammer M, Williams SCR, Parslow DM, Morgan MJ, Morris RG, Bullmore ET, Baron-Cohen S, Grey JA (2002) Functional magnetic resonance imaging of synesthesia: activation of V4/V8 by spoken words. Nat Neurosci 5(4):371375. doi: $10.1038 / \mathrm{nn} 818$

Ochsner KN, Ray RR, Hughes B, McRae K, Cooper JC, Weber J, Gabrieli JD, Gross JJ (2009) Bottom-up and topdown processes in emotion generation: common and distinct neural mechanisms. Psychol Sci 20(11):1322-1331. doi:10.1111/j.1467-9280.2009.02459.x

Olson IR, Plotzker A, Ezzyat Y (2007) The Enigmatic temporal pole: a review of findings on social and emotional processing. Brain 130(7):1718-1731. doi:10.1093/brain/awm052

Ramachandran VS, Hubbard EM (2001a) Psychophysical investigations into the neural basis of synaesthesia. Proc Biol Sci 268(1470):979-983. doi:10.1098/rspb.2000.1576

Ramachandran VS, Hubbard EM (2001b) Synaesthesia: a window into perception, thought and language. J. Conscious. Stud. $8(12): 3-34$

Rich A, Williams M, Puce A, Syngeniotis A, Howard M, McGlone F, Mattingley J (2006) Neural correlates of imagined and synaesthetic colours. Neuropsychologia 44(14):2918-2925. doi:10.1016/j.neuropsychologia.2006.06.024

Rothen N, Nyffeler T, von Wartburg R, Muri R, Meier B (2010) Parieto-occipital suppression eliminates implicit bidirectionality in grapheme-colour synaesthesia. Neuropsychologia 48(12):34823487. doi:10.1016/j.neuropsychologia.2010.07.032 
Rouw R, Scholte HS (2007) Increased structural connectivity in grapheme-color synesthesia. Nat Neurosci 10(6):792-797. doi: $10.1038 / \mathrm{nn} 1906$

Rouw R, Scholte HS (2010) Neural basis of individual differences in synesthetic experiences. J Neurosci 30(18):6205-6213. doi:10.15 23/jneurosci.3444-09.2010

Rubinov M, Sporns O, van Leeuwen C, Breakspear M (2009) Symbiotic relationship between brain structure and dynamics. BMC Neurosci 10(1):55. doi:10.1186/1471-2202-10-55

Shen S, Szameitat AJ, Sterr A (2007) VBM lesion detection depends on the normalization template: a study using simulated atrophy. Magn Reson Imaging 25(10):1385-1396. doi:S0730-725X(07)00231-7

Sidoroff-Dorso AV (2012) Investigación sobre la sinestesia en Rusia: las artes y la Ciencia. In: De Córdoba MJ, Riccó D (eds) Sinestesia. Los fundamentos teóricos, artísticos y científicos. Imprenta Del Carmen, Granada

Singh S, Modi S, Bagga D, Kaur P, Shankar LR, Khushu S (2012) Voxel-Based Morphometric Analysis in Hypothyroidism using DARTEL approach. J Neuroendocrinol. doi:10.1111/jne. 12001

Specht K, Laeng B (2011) An independent component analysis of fMRI data of grapheme-colour synaesthesia. J Neuropsychol 5(2):203-213. doi:10.1111/j.1748-6653.2011.02008.x

Spector F, Maurer D (2009) Synesthesia: a new approach to understanding the development of perception. Dev Psychol 45(1):175189. doi:10.1037/a0014171

Sperling JM, Prvulovic D, Linden DE, Singer W, Stirn A (2006) Neuronal correlates of colour-graphemic synaesthesia: a fMRI study. Cortex 42(2):295-303. doi:10.1016/S0010-9452(08)70355-1

Taylor KS, Seminowicz DA, Davis KD (2009) Two systems of resting state connectivity between the insula and cingulate cortex. Hum Brain Mapp 30(9):2731-2745. doi:10.1002/hbm.20705
Thompson E, Varela FJ (2001) Radical embodiment - neural dynamics and consciousness. Trends Cogn Sci 5(10):418-425

van Leeuwen TM, den Ouden HE, Hagoort P (2011) Effective connectivity determines the nature of subjective experience in grapheme-color synesthesia. J Neurosci 31(27):9879-9884. doi:10.152 3/JNEUROSCI.0569-11.2011

Ward J (2004) Emotionally mediated synaesthesia. Cogn Neuropsychol 21(7):761-772. doi:10.1080/02643290342000393

Weiss PH, Fink GR (2009) Grapheme-colour synaesthetes show increased grey matter volumes of parietal and fusiform cortex. Brain 132(1):65-70. doi:10.1093/brain/awn304

Weiss PH, Shah NJ, Toni I, Zilles K, Fink GR (2001) Associating colours with people: a case of chromatic-lexical synaesthesia. Cortex 37(5): 750-753

Weiss PH, Zilles K, Fink GR (2005) When visual perception causes feeling: enhanced cross-modal processing in grapheme-color synesthesia. Neuroimage 28(4):859-868. doi:10.1016/j.neuroim age.2005.06.052

Whitaker-Azmitia P (2010) Serotonin and development. In: Müller C, Jacobs B (eds) Handbook of behavioral neurobiology of serotonin vol 21. vol Handbook of behavioral neuroscience. Elsevier, pp 309-323. doi:10.1016/b978-0-12-374634-4.00003-4

Yamasaki H, LaBar KS, McCarthy G (2002) Dissociable prefrontal brain systems for attention and emotion. Proc Natl Acad Sci U S A 99(17):11447-11451. doi:10.1073/pnas 182176499182176499

Zeki S, Romaya JP (2008) Neural correlates of hate. PLoS ONE 3(10):e3556. doi:10.1371/journal.pone.0003556 